\title{
Protocol Development for Indoor Navigation using Bluetooth Device
}

\author{
Kumbhar Shrikant Sudhakar*, Birajdar Amar Dnyanoba and Prakash Ramchandran \\ School of Electronics Engineering Department, VIT University, Chennai - 600127, Tamil Nadu, India; \\ Kumbhar.shrikant2013@vit.ac.in, birajdar.amar2013@vit.ac.in, prakash.r@vit.ac.in
}

\begin{abstract}
Background: GPS based location measurement is not precise for indoor environment because satellite signals are getting attenuated and scattered by roof and wall. This can be achieved by a Bluetooth protocol developed for indoor navigation. Methods: In this paper an indoor navigation system using Bluetooth is proposed. Two Bluetooth devices used as positioning devices measure the Received Signal Strength (RSSI) of surrounding Bluetooth devices. Both the devices measure Received Signal Strength (RSSI) of a selected user device. These RSSI values are given to a raspberry Pi development board which acts as a processing unit in this system. In processing unit further processing is done by grid following method. There are three grids and nine points (spacing $1 \mathrm{~m}$ each) of grid following method finalized by performing repeated number of experiment. Findings: Raspberry Pi gives the location of surrounding device by comparing RSSI value obtained from grid following method during training phase and real time RSSI values obtained from two Bluetooth devices. This location is further plotted using GNUPLOT in raspberry Pi terminal and sent it to the user's smartphone. This graph file is transferred on user's smartphone by using USB Bluetooth dongle in a format of image.
\end{abstract}

Keywords: Bluetooth, Positioning System, Raspberry Pi, RSSI

\section{Introduction}

In present era, shopping experience is highly modernized due to development of modern gadgets. Shopping malls are getting attraction for its product quality, purity, plurality and variety. Shopping mall owner gives facilities such as security in mall, cleanness, interactive interior design and infrastructure ${ }^{1}$ for the comfort of customers. In addition, mall owner provides entertainment by arranging fun and entertainment program, fun spaces for kids. Big shopping malls are place of attraction for branded product manufacturers and used to display their new products. There is a requirement for the seller to give the updates of their new product to customer and customer want to know about the latest development. In this project a protocol is proposed to determine the proximity of a user and to send the new product information to the customer through Bluetooth. Present days number of smartphone users are increased in middle class families who are the main customers in shopping malls and this makes our communication method widely used.

GPS is inefficient for finding the position in indoor navigation. Because to determine the location using GPS three satellite signals are required in line of sight communication. These signals also get scattered or attenuated by internal structures such as roof, wall and other objects ${ }^{2}$.

The proposed system consists of two Bluetooth devices kept $2 \mathrm{~m}$ apart from each another ${ }^{3}$. This setup can place at the entrance of a shop. When customers are entering the shop one of the Bluetooth devices scan the smartphone and identified their mac address. By help of AT command Bluetooth device find the user-friendly Bluetooth name of smartphone. This name and relative information obtained during scanning such as mac address, class of device are stored for future use. When customer moves in shop two Bluetooth devices scan the RSSI of their smartphone $e^{4}$. In this case two Bluetooth devices scan same device. The current position of smartphone is found using an algorithm depending on these RSSI values ${ }^{5,6}$.

${ }^{*}$ Author for correspondence 
This paper is organized as follows. Section 2 gives the information about Raspberry Pi development board, used to implement our proposed work. Information about HC05 Bluetooth is explained in section 3 Section 4 gives information about DB9 connector which is required to connect HC05 Bluetooth and raspberry pi. Section 5 describes about cutecom software and section 6 describes AT-commands are used for controlling Bluetooth devices. Methodology is described in section 7 Final section describes results and conclusion.

\section{Raspberry Pi B+ Module}

Raspberry Pi is small credit card device, which is used for very high computation operations such as image processing, speech processing, signal processing and surveillance system, gaming and military applications ${ }^{7}$.

Some features of Raspberry Pi is given as follows.

- The Operating frequency is $700 \mathrm{MHz}$ with Broadcom BCM2835 CPU.

- The memory capacity is $512 \mathrm{MB}$ SDRAM.

- There are 40 pins for General Input Output (GPIO) operation.

- It provides full size High-Definition Multimedia Interface (HDMI).

- There are four ports and each specifies 2.0.

- There is Memory card slot where SD card is placed.

\section{HC-05 Bluetooth Module}

HC-05 Bluetooth model which is used for Serial Port Protocol (SPP). This can be configured in different modes such as master or slave depending upon application. SPP Bluetooth module have the specifications such as Bluetooth with V2.0+EDR (Enhanced Data Rate), 3Mbps Modulation with complete $2.4 \mathrm{GHz}$ radio transceiver and

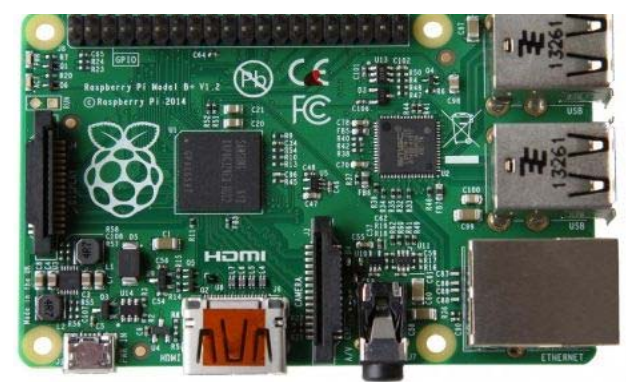

Figure 1. Raspberry Pi B+ development board. baseband. The size of this device is $12.7 \mathrm{~mm} \times 27 \mathrm{~mm}^{8}$. Figure 2 shows basic module with pin diagram of HC-05 Bluetooth. The HC-05 Bluetooth with RS232 is shown in Figure 3.

\subsection{Hardware Specification}

- The sensitivity of the device is $-80 \mathrm{dBm}$.

- The maximum transmission power is up to $+4 \mathrm{dBm}$.

- The required input output voltage is 1.8 to $3.6 \mathrm{~V}$.

- Programed Input/Output (PIO) control.

- UART interface present with programmable baud rate.

- This chip consists of an integrated antenna and edge connector.

\section{DB9 Connector}

It is a family of UART used for convert RS232 to USB serial port without modifying PCB. There are total six modules of DB9 connector, from this two operates on RS232 voltage and other four operates from $5 \mathrm{~V}$ to $3.3 \mathrm{~V}$. Where each is available in male or female format ${ }^{9}$. DB9 connector is shown in Figure 4.

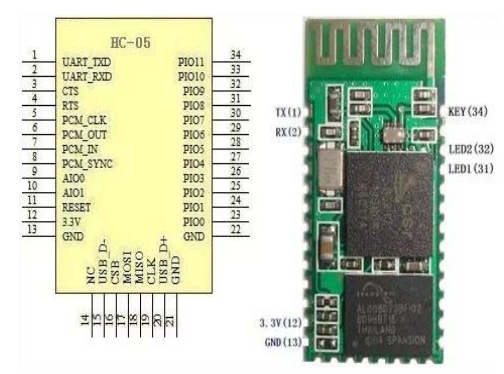

Figure 2. HC-05 basic model and pin diagram.

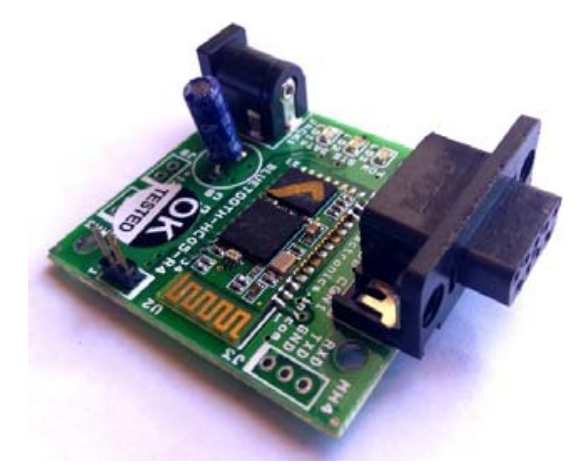

Figure 3. HC-05 Bluetooth with RS232. 


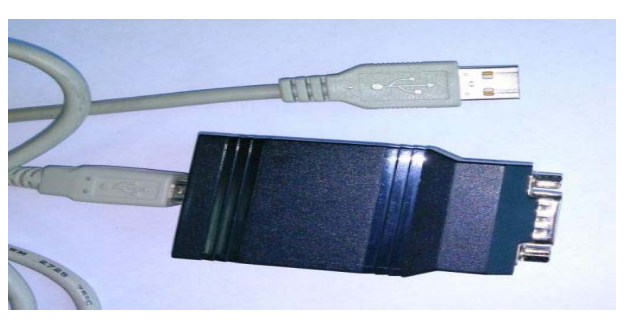

Figure 4. DB9 connector.

\section{Cutecom}

There are several software environments to access Bluetooth device using AT-command set. In windows environment Putty, Teraterm software is popularly used.

For Linux environment Minicom, Cutecom software are used widely. These are serial terminal software, used for displaying AT-command response of Bluetooth device. Features are as follows.

- Easy to use GUI.

- Line oriented interface instead of characteroriented.

- Easily differentiate input response and output response.

- It is configurable of various line feed character.

\section{AT-Command Set}

AT-commands are used for controlling modem, GSM, Bluetooth. AT is an abbreviation of attention. To give an instruction to device using AT command the instruction should start with 'AT' abbreviation. HC 05 Bluetooth is having very rich set of AT commands. In this paper for experiment purpose Cutecom software environment is used to access device using AT command ${ }^{10}$. List of AT-commands are as follows.
1. $\mathrm{AT}$
2. $\mathrm{AT}+\mathrm{ORGL}$
3. AT+RESET
4. $\mathrm{AT}+\mathrm{CLASS}=0$
5. $\mathrm{AT}+\mathrm{INIT}$
6. $\mathrm{AT}+\mathrm{INQM}=1,5,48$
7. AT+INQ
8. AT+BIND
9. $\mathrm{AT}+\mathrm{ADDR}$ ?
10. AT+ STATE?

\section{Methodology}

\subsection{Proposed Method for Indoor Navigation}

The proposed method consists of five steps. Two HC-05 Bluetooth devices inquire for surrounding Bluetooth devices with the help of AT-commands. The device address, class and RSSI values are present in inquiry response of surrounding Bluetooth devices. In second step these two HC-05 BT devices inquire for only selected users. The RSSI values are extracted from the results obtained through inquiry. This extracted RSSI values are analyzed in third step. Fourth step consists of location determining algorithm. This step gives location of surrounding device using RSSI values obtained from grid following algorithm and real time output RSSI values obtained from Bluetooth device. Output of previous step is graphically represented in fourth step. The graphical image obtained from fourth step is send to user's smart phone using USB Bluetooth dongle. The flow graph of Indoor navigation is shown in Figure 5.

The experimental setup for grid following is shown in Figure 6. Two Bluetooth devices (Device A and B) are separated by $2 \mathrm{~m}$ from each other. Fix the three grids and nine coordinate's points depending upon RSSI which are obtained from two Bluetooth devices. Here both devices access simultaneously. Each coordinate point is one meter from another point.

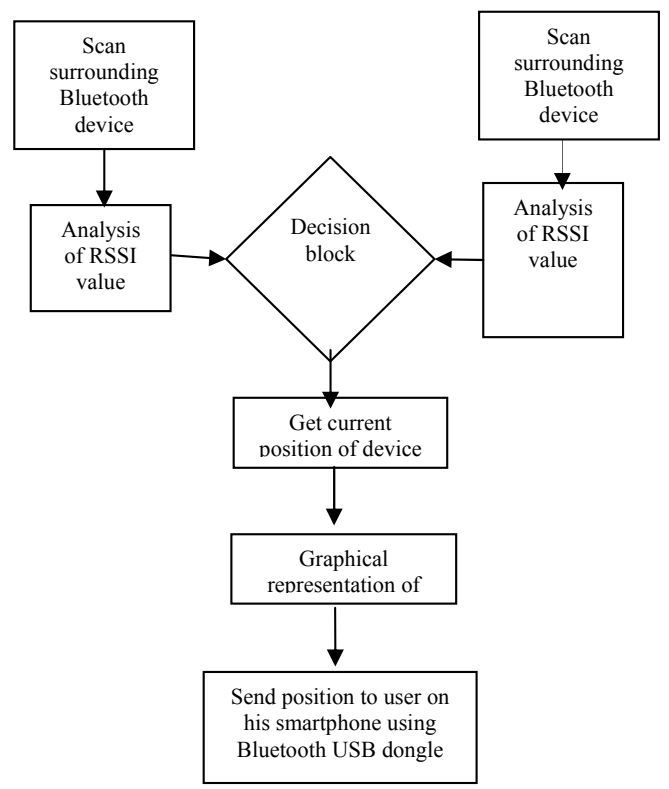

Figure 5. Flow chart of indoor navigation. 


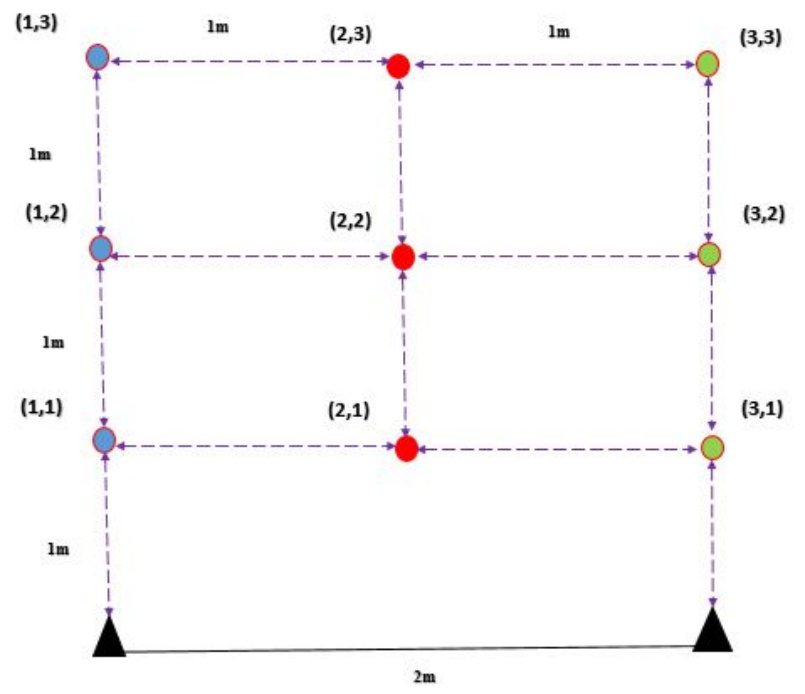

Figure 6. Grid following method.

\subsection{Results}

For implementation of proposed method two HC 05 Bluetooth devices are used. These devices are placed $2 \mathrm{~m}$ apart from each other. These two HC-05 Bluetooth devices are simultaneously operated using the Raspberry pi board. For experimental purpose a laptop is used as display device. Grid points of grid following method are highlighted in Figure 7. For experimental purpose smartphone is placed near grid points. The decision block locate correct position of the smartphone. Stepwise analysis of flow for indoor navigation is done in following steps. Experimental setup shown in Figure 7.

Step 1: The inquiry of the surrounding Bluetooth devices is performed by two HC-05 Bluetooth devices. These HC-05 Bluetooth devices are interfaced to raspberry pi with the help of DB9 connector. The DB9 connector converts RS232 serial terminal to USB terminal. The initial setting consists of changing the HC-05device data transfer mode into AT-command mode. First command given is 'AT' then the device will respond 'OK'. The default mode of the Bluetooth device is the slave mode and set is to master mode. In master mode the Bluetooth device will inquire the surrounding devices. This procedure is conducted using cutecom software. The automated system is further implemented using shell scripting.

Step 2: In this step RSSI values are analyzed which are obtained from real time inquiry of HC-05

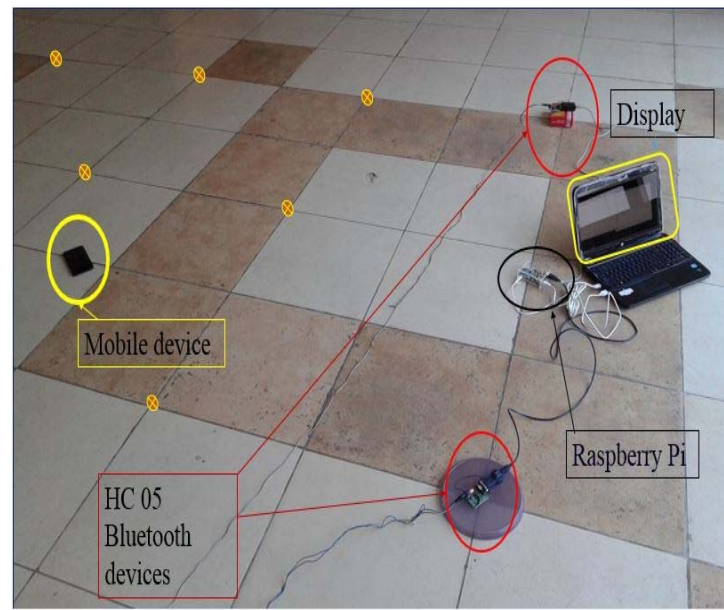

Figure 7. Experimental Setup.

Bluetooth device. Process flow graph of extracting RSSI value from Bluetooth inquiry output is shown in Figure 8. Initially class is extracted of selected user device from inquiry result. In next this class is set for both Bluetooth devices so that it can inquire for the selected user only. Then an inquiry is done for selected user and from the inquiry result the RSSI value is extracted. This RSSI value in hexadecimal converted to a weighted decimal. This output is given to the decision block.

Step 3: The correct position of user is located by using Grid following method and the real time output obtained from second step. The graphical representation of grid following method by using GNUPLOT is shown in Figure 9. There are total nine grid points and each point separated from other point with a distance of 1 metre. The bluetooth devices are placesed at co-ordinate points $(1,0)$ and $(3,0)$. Othere nine poits are represented by dots and which are located on three grids.

Step 4: The obtained location is graphically plotted using GNUPLOT as shown in Figure 10.

The final software result simulation is shown in Figure 11. Each device gives inquiry responses for five times. This response string consists of address, class and RSSI value of particular device. In the following step RSSI value is extracted from the output string. It gives average of these five points which are takenfrom both BT devices. Software simulation shows the obatined average values are -75 and -69 respectively which are from raspberry Pi. These values are given to the decision block and it locates the position of user.The located position is represented in 


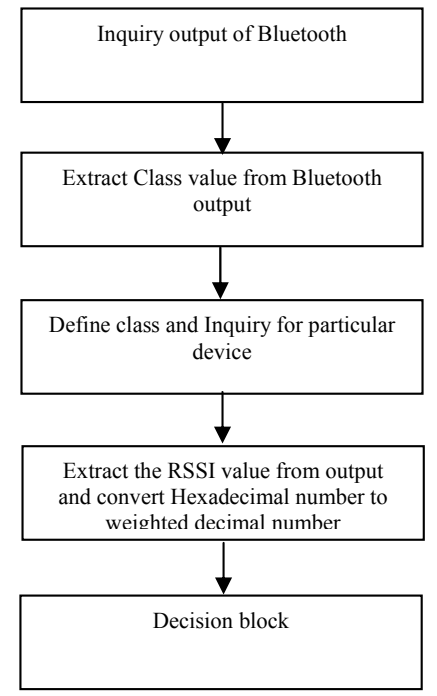

Figure 8. Extracting RSSI value from output.

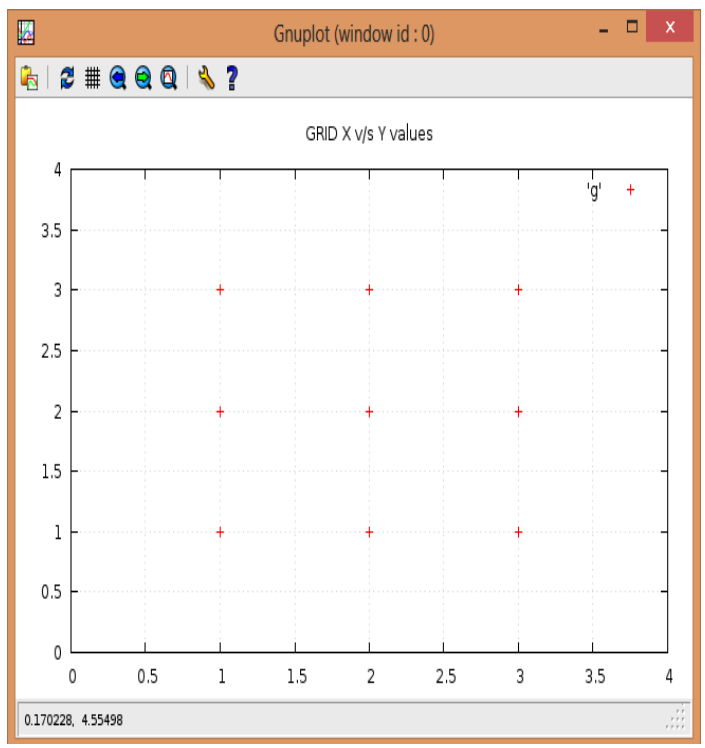

Figure 9. Representation of grid in Gnu plot.

graphical method. In this experiment the location of user is following first geid and near co-ordinate point $(1,2)$. Graphical result is ploted on GNUPLOT tool. The location of user is heilighted by a circle and other grid poits are heilighted by dots.

\section{Conclusion}

In this paper consider a Bluetooth based positioning with a grid following method to locate the position of a smartphone. The RSSI values of the smartphone is measured with

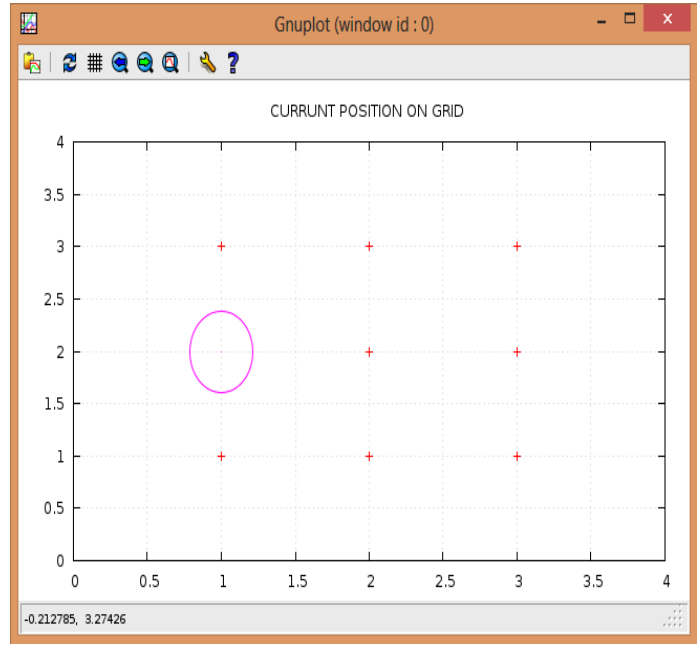

Figure 10. Located position of the smartphone.

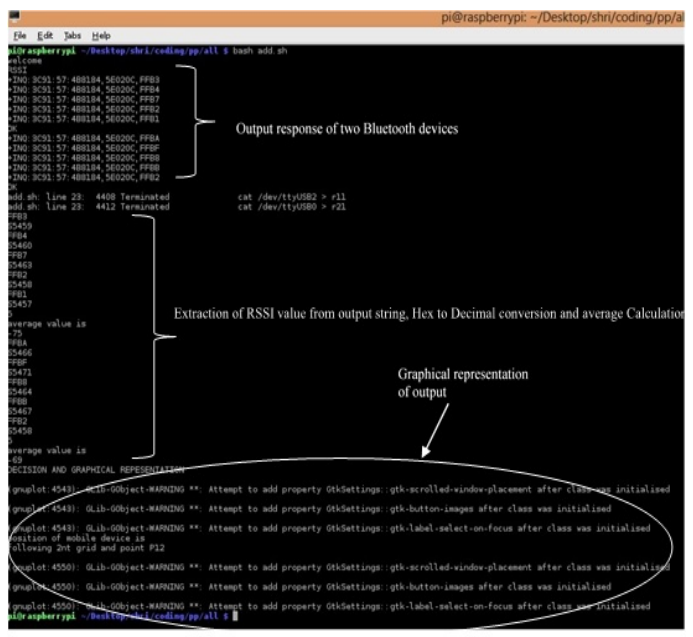

Figure 11. Software result.

respect to two Bluetooth devices. From inquiry output of Bluetooth device extract the required RSSI value properly and it converted into suitable number format. Then these values are given to decision block. By using grid following method the location of the smart phone in the grid system is found. This position is graphically represented using GNUPLOT software. This enable us to send a relevant information with respect to the position of user.

\section{References}

1. El-Adly MI. Shopping Mall Attractiveness: A Segmentation Approach. International Journal of Retail and Distribution Management. 35(11): 936-50. 
2. Yih-Guang J, Hsien-Wei T, Yang-Han L, Chih-Yuan L, Liang-Yu Y. Accurate Bluetooth Positioning Using Weighting and Large Number of Devices Measurements. Wireless Pers Communication. 2014; 79:1129-43.

3. Zhou S, Pollard JK. Position Measurement using Bluetooth. IEEE Transactions on Consumer 556 Electronics. 2006 May; 52(2).

4. Subhan F, Hasbullah H, Rozyyev A, Bakhsh ST. Analysis of Bluetooth signal parameters for indoor positioning systems. IEEE International Conference on Computer and Information Science (ICCIS); USA: IEEE Computer Society; 2012. p. 151-6.

5. Hossain AKM, Soh WS. A comprehensive study of Bluetooth signal parameter for location. IEEE International Symposium on Personal, Indoor and Mobile Radio Communications (PIMRC’07). 2007.
6. Yapeng $\mathrm{W}, \mathrm{Xu} \mathrm{Y,} \mathrm{Yutian} \mathrm{Z,} \mathrm{Yue} \mathrm{L,} \mathrm{Laurie} \mathrm{C.} \mathrm{Bluetooth}$ Positioning using RSSI and Triangulation Methods. IEEE $10^{\text {th }}$ Consumer Communications and Networking Conference (CCNC); 2013.

7. The Raspberry Pi - Single Board Computer.

8. HC Serial Bluetooth Products User Instructional Manual datasheet.

9. Future Technology Devices International Ltd. The DB9-USB Family of UART Converter Modules datasheet Document Reference No.: FT_000204 Version 2.0 Issue Date: 201108-31.

10. HC-03/05 Embedded Bluetooth Serial Communication Module AT command set datasheet Last revised: April, 2011. 